\section{Estimativas de insegurança alimentar grave nos municípios brasileiros}

\author{
Estimates of severe food insecurity \\ in Brazilian municipalities
}

\author{
1 Faculdade de Ciências \\ da Saúde, Universidade de \\ Brasília, Brasília, Brasil. \\ 2 Faculdade de Saúde \\ Pública, Universidade de São \\ Paulo, São Paulo, Brasil. \\ Correspondência \\ M. B. Gubert \\ Departamento de Nutrição, \\ Faculdade de Ciências da \\ Saúde, Universidade de \\ Brasília. \\ Campus Universitário Darcy \\ Ribeiro, Brasília, $D F$ \\ 70910-900, Brasil. \\ muriel@unb.com.br
}

\begin{abstract}
Prevalence of severe food insecurity was estimated for Brazilian municipalities based on the 2004 National Household Sample Survey (PNAD). First, a logistic regression model was developed and tested with this database. The model was then applied to the 2000 census data, generating severe food insecurity estimates for the Brazilian municipalities, which were subsequently analyzed according to the proportion of families exposed to severe food insecurity. Severe food insecurity was mainly concentrated in the North and Northeast regions, where $46.1 \%$ and $65.3 \%$ of municipalities showed high prevalence of severe food insecurity, respectively. Most municipalities in the Central West region showed intermediate prevalence of severe food insecurity. There was wide intra-regional variation in severe food insecurity, while the South of Brazil showed the most uniform distribution. In conclusion, Brazil displays wide inter and intra-regional variations in the occurrence of severe food insecurity. Such variations should be identified and analyzed in order to plan appropriate public policies.
\end{abstract}

Hunger; Forecasting; Cities; Public Health Nutrition
Muriel Bauermann Gubert 1 Maria Helena D’Aquino Benício 2 Leonor Maria Pacheco dos Santos 1

\section{Introdução}

Observa-se no Brasil uma preocupação crescente com as condições de segurança alimentar e nutricional da população ${ }^{1}$. Essa temática, introduzida na pauta política do país pelo sanitarista Josué de Castro na década de 40 2, hoje assume especial importância pela priorização do governo no combate à fome e à miséria.

A segurança alimentar e nutricional é, sem dúvida, um processo complexo e de difícil aferição. O conceito adotado pelo Brasil, conforme a Lei $n^{\circ} .11 .346$ de 2006, caracteriza a segurança alimentar e nutricional como um processo multidimensional, que envolve toda a cadeia alimentar, sendo determinado por fatores que vão desde a produção agrícola, distribuição e acesso dos alimentos à população, acesso econômico ao alimento, até a própria escolha alimentar, baseada em componentes culturais 3 .

Até pouco tempo, os dados sobre a prevalência de insegurança alimentar no país eram limitados. A maioria das estimativas baseava-se apenas em indicadores indiretos do processo, tais como renda, estado nutricional da população e disponibilidade alimentar domiciliar. Ressalta-se que cada um desses indicadores mensura uma parte da problemática da segurança alimentar e nutricional, sendo complementares uns aos outros 4 . Recentemente, outro instrumento foi agregado ao cabedal de opções de aferição de insegurança alimentar, a Escala Brasileira de Insegurança Ali- 
mentar (EBIA) 5. A EBIA caracteriza-se por avaliar a situação de insegurança alimentar familiar, incluindo tanto a percepção das pessoas ${ }^{6}$ como outras questões bem objetivas, visando a avaliar a disponibilidade de alimentos no domicílio, e vêm preencher algumas lacunas deixadas pelos outros métodos 4 .

A EBIA foi aplicada na Pesquisa Nacional por Amostra de Domicílios (PNAD) 5, no ano de 2004. Essa pesquisa, de base domiciliar, forneceu dados confiáveis sobre a prevalência de insegurança alimentar para o Brasil e suas Unidades da Federação (UF) e Distrito Federal, tanto para a zona rural quanto para a urbana. A metodologia da PNAD, porém, só permite a desagregação de dados em nível estadual, deixando desta forma, o município - unidade básica da organização política do país e, portanto, ator primordial das políticas públicas do setor social 7 - sem informações sobre a ocorrência deste problema entre seus habitantes.

O Brasil é um país de desigualdades, expressas nas esferas nacional, estadual e municipal 8 . Dados municipais de prevalência de insegurança alimentar são, portanto, necessários à execução da gestão pública, pois existem importantes discrepâncias sociais e econômicas intra-estaduais que se manifestam nos municípios. Sabendose que a insegurança alimentar é parcialmente oriunda desses determinantes, esperam-se diferenças importantes na sua ocorrência em nível municipal. Isso pode ser observado por pesquisa realizada na Paraíba, em dez municípios, onde a insegurança alimentar grave variou entre $5,4 \%$ e $22,8 \% 9$

Dessa forma, o presente estudo tem por objetivo gerar estimativas de prevalência da insegurança alimentar grave para todos os municípios brasileiros, revelando a magnitude e distribuição de tão importante problema no país.

\section{Métodos}

A EBIA, aplicada na PNAD 2004, é composta de quinze perguntas e gera um escore que classifica a insegurança alimentar domiciliar em três níveis: leve, moderado e grave. Neste estudo optou-se por pesquisar a ocorrência de insegurança alimentar grave que, segundo a metodologia da EBIA, caracteriza a ocorrência do fenômeno da fome nos domicílios estudados, nos últimos três meses, tempo de referência da pesquisa 10 .

No presente estudo foi aplicado o método desenvolvido por Elbers et al. ${ }^{11}$ para estimar pobreza e desigualdade em estudos de mapeamento da pobreza em diversos países, e prevê o estabelecimento de modelo preditivo baseando-se em inquérito com representatividade nacional e posterior aplicação do modelo aos dados do censo populacional do país em questão.

As estimativas da prevalência da insegurança alimentar grave nos municípios brasileiros foram calculadas tendo como base modelo estatístico de predição de insegurança alimentar grave domiciliar proposto por Gubert 12, segundo metodologia de Elbers et al. 11, e descrito a seguir. Os domicílios estudados (111.922) foram categorizados dicotomicamente em inseguros (quando apresentavam insegurança grave) ou seguros. Para a seleção das variáveis preditivas considerouse sua disponibilidade e similaridade de aferição na PNAD 2004 e na amostra do Censo Demográfico 2000 (http://www.ibge.gov.br), com vistas à utilização posterior do modelo para a predição da insegurança alimentar grave nos municípios brasileiros. Inicialmente, foi realizada análise de regressão logística univariada de cada uma das potenciais variáveis preditivas com a variável resposta. Dentre as variáveis disponibilizadas pela PNAD, priorizou-se aquelas tradicionalmente relacionadas com a ocorrência de insegurança alimentar. As variáveis que apresentaram $\mathrm{p}<0,05$ no teste de Wald foram incluídas no processo de modelagem. A modelagem foi realizada por meio de regressão logística multivariada, sendo incluídas primeiramente as variáveis com maior relação com insegurança alimentar. Permaneceram no modelo final aquelas que foram estatisticamente significativas $(\mathrm{p}<0,05)$.

Durante a elaboração foram testados modelos distintos para as regiões brasileiras e para zonas urbanas e rurais. Entretanto, a aplicação de equações distintas não melhorava o poder de predição do modelo. Optou-se, então, por um modelo único nacional, sendo incluídas todas as UF, mesmo que as mesmas não se apresentassem significativas. Um modelo único já havia sido utilizado em estudos anteriores no Brasil, com sucesso 13,14.

O modelo estatístico gerado permitiu estimar a probabilidade da ocorrência de insegurança alimentar grave em um determinado domicílio, em função da presença ou ausência de fatores preditivos. As variáveis incluídas no modelo, determinantes da insegurança alimentar grave, foram: renda per capita; escolaridade; cor e sexo do chefe do domicílio; situação urbana ou rural; presença de água encanada; presença de crianças; total de moradores e estado da federação. A equação do modelo resultante é: 
$\log \mathrm{p} / 1-\mathrm{p}=-5,171+2,428[$ menor $1 / 4$ SMPC] $+1,575[\geq 1 / 4 \mathrm{a}<1 / 2 \mathrm{SMPC}]+0,813[\geq 1 / 2 \mathrm{a}<1$ SMPC $]+1,561[$ menos 1 ano estudo $]+1,214[1$ a 3 anos estudo] + 0,966[4 a 7 anos estudo] + 0,723 [7 a 10 anos estudo] $+0,402$ [preto/pardo] + 0,471 [mulher] + 0,576[sem água] - 0,685[rural] + 0,452 [três ou mais crianças] $+1,149[\mathrm{RR}]+$ $0,997[\mathrm{AP}]+0,886[\mathrm{RN}]+0,846[\mathrm{PA}]+0,841[\mathrm{~PB}]+$ $0,737[\mathrm{MA}]+0,693[\mathrm{AM}]+0,681[\mathrm{AC}]+0,624[\mathrm{CE}]+$ $0,610[\mathrm{BA}]+0,517[\mathrm{RS}]+0,396[\mathrm{PE}]+0,335[\mathrm{TO}]+$ $0,297[\mathrm{GO}]+0,291[\mathrm{MS}]+0,272[\mathrm{RJ}]+0,261[\mathrm{SP}]+$ $0,248[\mathrm{PR}]+0,187[\mathrm{PI}]+0,177[\mathrm{DF}]+0,117[\mathrm{MG}]+$ $0,084[\mathrm{MT}]+0,067[\mathrm{ES}]+0,024[\mathrm{AL}]-0,147[\mathrm{RO}]-$ $0,596[\mathrm{SE}]-0,079$ [total de moradores].

O modelo utilizado para a estimativa de insegurança alimentar grave teve sua adequação avaliada pelos testes de Hosmer-Lemeshow ( $\mathrm{p}=$ 0,561 ) e curva ROC (área $=0,823$ ), obtendo valores considerados satisfatórios, o que determina um adequado poder de predição, possibilitando seu uso para a determinação de insegurança alimentar grave domiciliar nos municípios brasileiros 12 .

Uma vez determinada a equação, empregouse metodologia semelhante à utilizada por Benicio \& Monteiro ${ }^{15}$ para obtenção das prevalências municipais. O modelo foi aplicado aos microdados da amostra do Censo Demográfico 2000, realizado pelo Instituto Brasileiro de Geografia e Estatística (IBGE), e de domínio público. A escolha da amostra do censo como base empírica devese ao fato de esta ser a única pesquisa disponível que fornece dados representativos municipais.

As variáveis da amostra do Censo Demográfico foram então categorizadas de forma similar a das variáveis preditivas do modelo, para viabilizar a aplicação da equação do modelo a esse banco de dados.

Com a aplicação da equação à amostra do censo obteve-se a probabilidade de ocorrência da insegurança alimentar grave em cada domicílio estudado pela amostra do Censo Demográfico 2000. As médias dessas probabilidades em cada município permitiram estimar a prevalência de insegurança alimentar grave para cada um dos 5.507 municípios brasileiros da malha municipal de 2000 .

Como as prevalências de insegurança alimentar grave nacionais mais recentes são oriundas da PNAD do ano de 2004, optou-se por corrigir as prevalências municipais estimadas de 2000 (a partir do modelo) para o ano de 2004, mesmo ano da PNAD. Para tal, multiplicou-se as estimativas municipais de insegurança alimentar grave calculadas por um fator de correção, definido como a razão entre as prevalências estaduais de insegurança alimentar grave aferidas pela PNAD 2004 e as estimadas para o ano 2000 para as UF brasileiras com base no modelo.
A utilização desse método para gerar estimativas tem algumas limitações, como trabalhar com um número restrito de variáveis, pois devem estar presentes e disponíveis nas mesmas categorias em ambas as pesquisas utilizadas (PNAD 2004 e Censo Demográfico 2000) e também o fato de a geração da estimativa só ser possível para a malha municipal de 2000, ano do censo.

Neste artigo, as prevalências municipais geradas não serão apresentadas individualmente e sim agrupadas por UF, priorizando-se a análise da distribuição da insegurança alimentar no Brasil.

Com o objetivo de facilitar a descrição da distribuição espacial da insegurança alimentar grave no país, optou-se por apresentar os resultados segundo uma divisão categórica em quartis de prevalência de insegurança alimentar. Dessa forma, os municípios foram categorizados em 4 níveis de prevalência de insegurança alimentar: baixa (até $3,6 \%)$; média $(3,7 \%$ a $5,4 \%)$; alta $(5,5 \%$ a $12,7 \%)$; e muito alta $(12,8 \%$ a $31,1 \%)$.

Os processamentos foram realizados utilizando-se o programa SPSS, versão 15.0 paraWindows (SPSS Inc., Chicago, Estados Unidos) e a aplicação da equação ao censo foi realizada com o programa Stata (Stata Corp., College Station, Estados Unidos). Em todas as etapas da modelagem foi considerada a estrutura complexa das amostras e utilizados os pesos amostrais recomendados pelo IBGE para as análises de ambas as pesquisas 16,17 .

\section{Resultados}

Foram estimadas as prevalências municipais dos 5.507 municípios brasileiros para o ano de 2004, conforme a malha municipal vigente no ano do Censo Demográfico. A prevalência de insegurança alimentar grave estimada pelo modelo para o Brasil, no ano de 2004, com base nas estimativas municipais, foi de $8,3 \%$ (Tabela 1 ). As estimativas para o conjunto dos municípios brasileiros confirmam a grande heterogeneidade que caracteriza a distribuição da freqüência da insegurança alimentar grave no país.

A região com a maior ocorrência de insegurança alimentar grave foi a Região Nordeste, onde a prevalência média municipal foi de $14,5 \%$, contra 3,6\% na Região Sul, a de menor prevalência. Analisando-se a variação intra-regional na ocorrência de insegurança alimentar grave, observa-se que a Região Sul é a mais uniforme, uma vez que as prevalências de insegurança alimentar grave variaram apenas 7,2 pontos percentuais entre seus municípios. Ao contrário, a Região Nordeste revelou-se muito díspare, com 
Prevalência de insegurança alimentar grave em municípios brasileiros segundo região. Brasil, 2004.

\begin{tabular}{lcccc}
\hline Região & Municípios (n) & $\begin{array}{c}\text { Insegurança alimentar } \\
\text { grave (\%) }\end{array}$ & $\begin{array}{c}\text { Mínimo de insegurança } \\
\text { alimentar grave } \\
\text { municipal (\%) }\end{array}$ & $\begin{array}{c}\text { Máximo de insegurança } \\
\text { alimentar grave } \\
\text { municipal (\%) }\end{array}$ \\
\hline Norte & 449 & 12,3 & 2,7 & 28,2 \\
Nordeste & 1.787 & 14,5 & 2,1 & 31,1 \\
Sudeste & 1.666 & 4,6 & 1,7 & 13,2 \\
Sul & 1.159 & 3,6 & 1,0 & 8,2 \\
Centro-oeste & 446 & 5,4 & 2,1 & 12,6 \\
Total & 5.507 & 8,3 & 1,0 & 31,1 \\
\hline
\end{tabular}

variação municipal na prevalência de insegurança alimentar grave entre $2,1 \%$ e $31,1 \%$ (Tabela 1 ).

Uma intensa variação intra-estadual também é encontrada na maioria das UF brasileiras, a exemplo das variações regionais observadas. A Figura 1 apresenta as médias de prevalências municipais de insegurança alimentar grave para cada UF e os valores mínimos e máximos destas prevalências. Observa-se que Rondônia, Sergipe e Santa Catarina apresentam menores variações intra-estaduais (aproximadamente 3,8 pontos percentuais). A maior variação em um mesmo estado ocorreu no Maranhão, onde o município com menor prevalência apresentava $6,9 \%$ de insegurança alimentar grave contra $31,1 \%$ do município com maior prevalência.

Analisando-se o componente municipal isoladamente, observa-se grande intervalo de variação. No Brasil, existem municípios onde a insegurança alimentar grave acomete $1 \%$ dos domicílios, até municípios onde a mesma freqüência atinge aproximadamente $31 \%$ dos domicílios.

Com a finalidade de melhor observar a distribuição espacial da insegurança alimentar grave no território brasileiro, foi elaborado o mapa do país, apresentado na Figura 2. Nele, estão representadas por cores mais claras as áreas dos municípios onde é mais baixa a prevalência da insegurança alimentar grave. Essa tonalidade vai ficando mais intensa à medida que a prevalência de insegurança alimentar grave aumenta. Analisando-se essa distribuição nas regiões do país, observa-se que existe uma tendência nítida de maior concentração de municípios com prevalências de insegurança alimentar grave elevadas nas regiões Norte e Nordeste, contrastando com percentuais mais tênues nas regiões Sul e Sudeste.

A Tabela 2 apresenta a distribuição de insegurança alimentar grave dos municípios brasileiros em quartis, segundo a região e UF. Observa-se que na Região Nordeste a maior parte dos municípios $(65,6 \%)$ apresenta prevalências muito altas de insegurança alimentar grave, o mesmo ocorrendo na Região Norte, onde 46,1\% dos municípios estão nesta categoria. Situação muito diversa da apresentada pelas regiões Sul e Centro-oeste, onde nenhum município apresentou prevalência muito alta de insegurança alimentar grave. As regiões com menores freqüências municipais de insegurança alimentar grave foram as Sul e Sudeste. No sul do país a maior parte dos municípios (52,8\%) apresentou prevalências de insegurança alimentar grave baixas. A maior parte dos municípios da Região Sudeste encontravase, também, nas categorias de menor prevalência de insegurança alimentar grave.

O mesmo fenômeno de heterogeneidade regional ocorre em nível estadual. Na Região Norte, a maioria dos municípios apresenta prevalências muito altas $(46,1 \%)$ ou altas $(41,9 \%)$ de insegurança alimentar grave. O estado que apresentou situação mais adversa foi Roraima, onde todos os municípios apresentaram prevalências muito altas de insegurança alimentar grave. Situação igualmente preocupante é apresentada pelos estados do Acre, Pará e Amapá, onde mais de $80 \%$ dos municípios apresentam percentuais muito altos de insegurança alimentar grave. $\mathrm{Na}$ região, a situação mais favorável foi apresentada pelo Estado de Rondônia, onde 94,2\% dos municípios apresentaram prevalências baixas e médias.

A Região Nordeste apresenta a pior situação do país. Nela, predominam os municípios com prevalência muito alta de insegurança alimentar grave $(65,6 \%)$, seguidos de municípios com prevalências altas (30,3\%). Dentre os estados que compõem a região, a pior situação é apresentada pelo Maranhão, onde 97,7\% dos municípios 
Variação estadual (em ordem crescente) e média da prevalência de insegurança alimentar grave em municípios brasileiros. Brasil, 2004

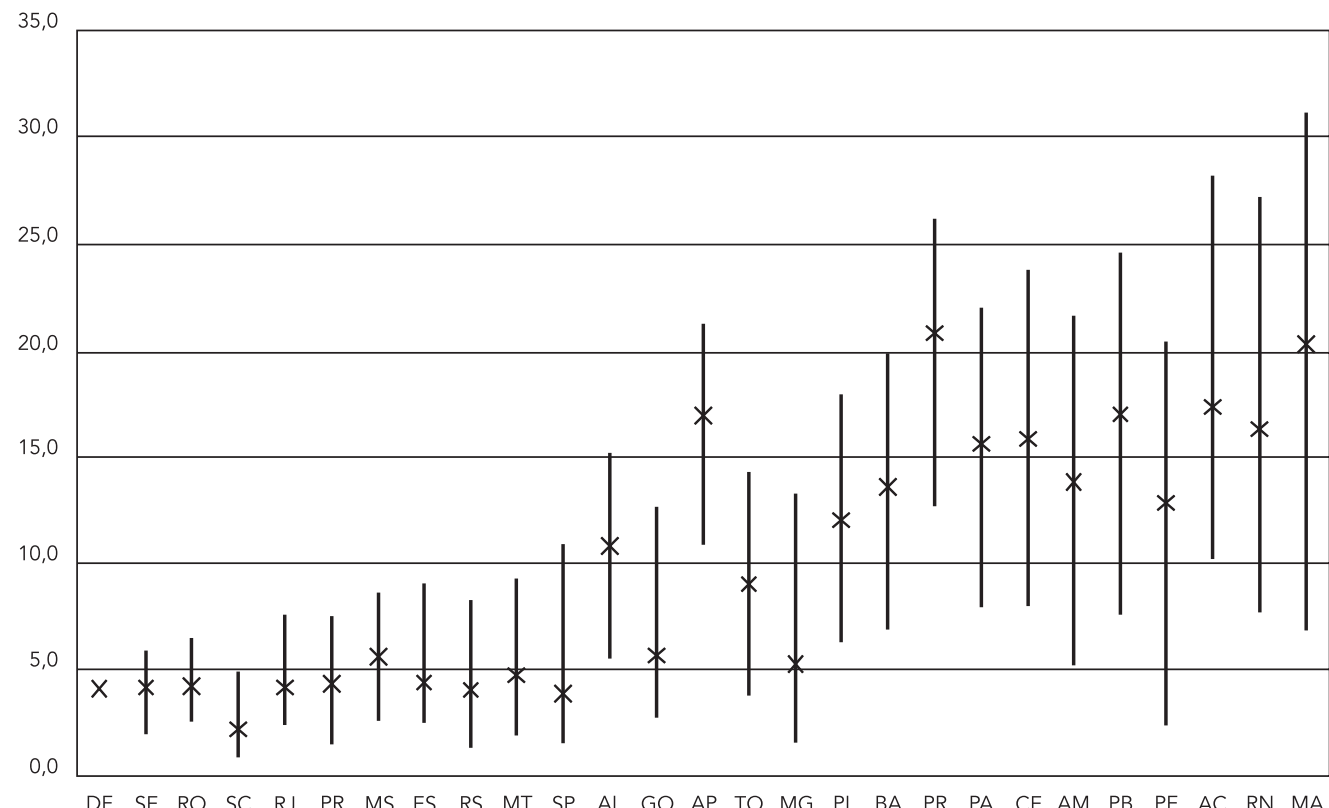

AC: Acre; AL: Alagoas; AM: Amazonas; AP: Amapá; BA: Bahia; CE: Ceará; DF: Distrito Federal; ES: Espírito Santo; GO: Goiás; MA: Maranhão; MG: Minas Gerais; MS: Mato Grosso do Sul; MT: Mato Grosso; PA: Pará; PB: Paraíba; PE: Pernambuco; PI: Piauí; PR: Paraná; RJ: Rio de Janeiro; RN: Rio Grande do Norte; RO: Rondônia; RR: Roraima; RS: Rio Grande do Sul; SC: Santa Catarina; SE: Sergipe; SP: São Paulo; TO: Tocantins.

apresentam prevalência muito alta de insegurança alimentar grave, seguido de perto pela Paraíba, onde 93,7\% dos municípios apresentam a mesma situação. Na região, apenas os estados de Pernambuco e Sergipe possuem municípios com baixas prevalências de insegurança alimentar grave, sendo que o primeiro apresenta apenas um município nesta situação, o que representa apenas 0,5\% do total de municípios deste estado. No Nordeste, a situação mais favorável é apresentada por Sergipe, onde não existem municípios com prevalências muito altas, pois $96 \%$ estão nas categorias de baixa e média prevalências de insegurança alimentar grave.

Diferentemente do que ocorreu nas regiões Norte e Nordeste, predominam na Região Sudeste municípios com baixa $(40,1 \%)$ e média $(38,4 \%)$ exposição à insegurança alimentar grave. Excetuando-se o Estado de Minas Gerais, nenhum outro apresenta municípios com prevalência muito alta de insegurança alimentar grave. A situação mais favorável é a do Estado de São Paulo, que tem mais da metade $(50,5 \%)$ de seus municípios com prevalências baixas de insegurança alimentar grave.

Predominam na Região Centro-oeste os municípios com prevalências médias de insegurança alimentar grave (50,4\%). A situação em relação à baixa ocorrência de insegurança alimentar é menos favorável que na Região Sudeste, uma vez que apenas $11 \%$ dos municípios da Região Centro-oeste encontram-se nesta situação. Entretanto, inexistem na região municípios com exposição grave à insegurança alimentar grave. Na região, a melhor situação é apresentada pelo Estado do Mato Grosso, onde 23,8\% dos municípios apresentam baixa prevalência de insegurança alimentar grave. O Distrito Federal apresenta média exposição à insegurança alimentar grave.

Dentre as cinco regiões brasileiras, a Região Sul é aquela que apresenta situação de insegurança alimentar grave mais favorável. A maioria dos municípios da Região Sul encontra-se na 


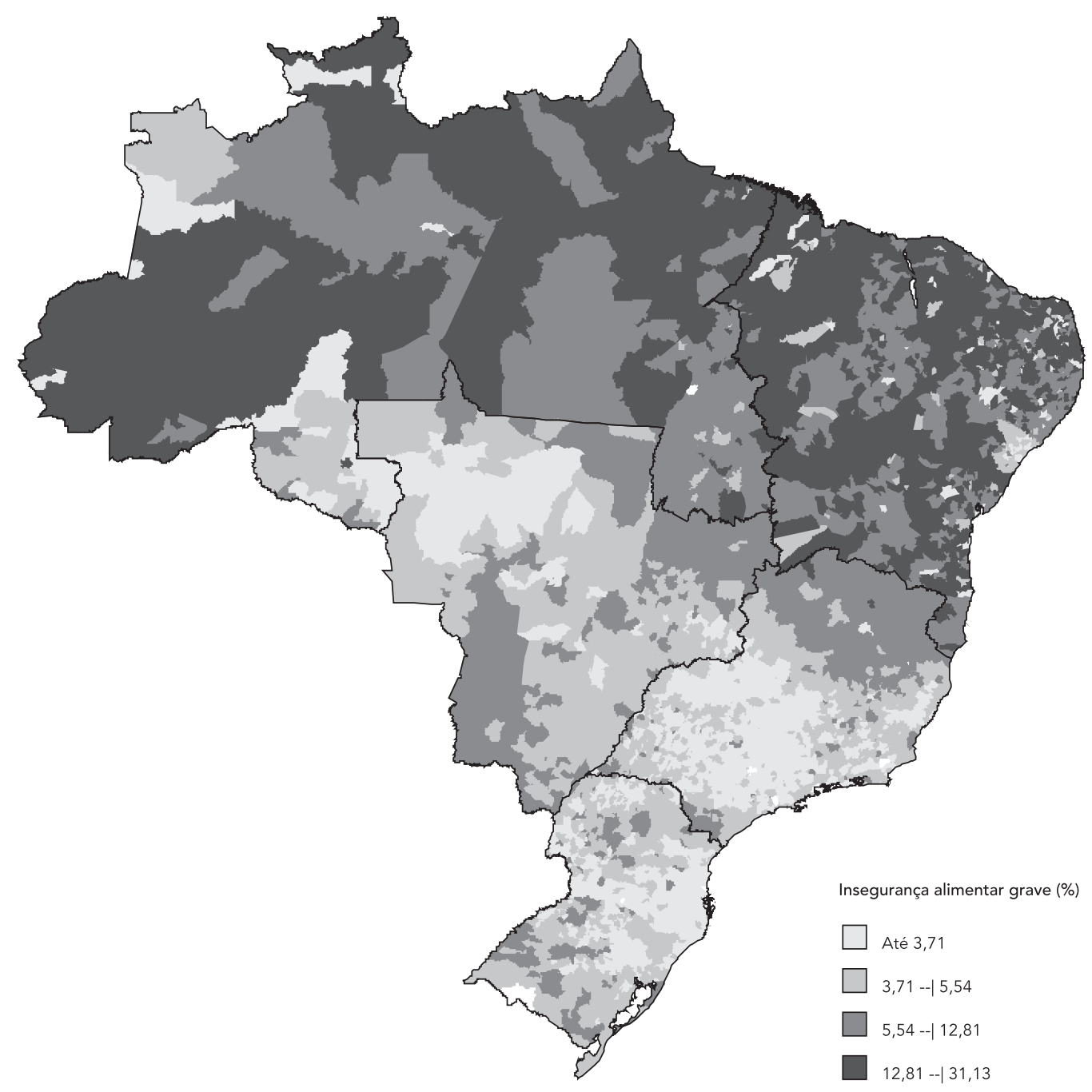

categoria de baixa prevalência de insegurança alimentar grave (52,8\%), não existem municípios expostos a prevalências muito altas e apenas $10 \%$ dos municípios da região têm prevalências consideradas altas de insegurança alimentar grave. Santa Catarina destaca-se como o estado em melhor situação, uma vez que 95,6\% de seus municípios têm baixas prevalências de insegurança alimentar grave e nenhum é classificado como exposto a uma prevalência alta ou muito alta de insegurança alimentar grave. Além de Santa Catarina, o Rio Grande do Sul e o Paraná apresentam os melhores resultados, nesta ordem.

\section{Discussão}

Neste estudo evidenciou-se que a distribuição da insegurança alimentar no Brasil é heterogênea, apresentando os municípios percentuais muito distintos de insegurança alimentar em uma mesma região ou até mesmo em uma mesma UF. Essa análise em nível municipal ainda não havia sido evidenciada anteriormente para todo o território nacional. Apenas o estudo de Viana \& Segall-Côrrea 9 havia analisado municípios no interior do Estado da Paraíba, detectando, também, prevalências discrepantes de insegu- 
Categorias de prevalência de insegurança alimentar em municípios segundo Unidades da Federação. Brasil, 2004.

\begin{tabular}{|c|c|c|c|c|c|c|c|c|c|c|}
\hline \multirow[t]{4}{*}{ Região/Estados } & \multicolumn{10}{|c|}{ Prevalência municipal de insegurança alimentar grave } \\
\hline & \multirow{2}{*}{\multicolumn{2}{|c|}{$\begin{array}{c}\text { Baixa } \\
\text { (até } 3,6 \% \text { ) }\end{array}$}} & \multirow{2}{*}{\multicolumn{2}{|c|}{$\begin{array}{l}\text { Média } \\
(3,7-5,4 \%)\end{array}$}} & \multirow{2}{*}{\multicolumn{2}{|c|}{$\begin{array}{c}\text { Alta } \\
(5,5-12,7 \%)\end{array}$}} & \multirow{2}{*}{\multicolumn{2}{|c|}{$\begin{array}{l}\text { Muito alta } \\
(12,8-31,1 \%)\end{array}$}} & \multirow{2}{*}{\multicolumn{2}{|c|}{ Total }} \\
\hline & & & & & & & & & & \\
\hline & $\mathrm{n}$ & $\%$ & $\mathbf{n}$ & $\%$ & $\mathbf{n}$ & $\%$ & n & $\%$ & $\mathbf{n}$ & $\%$ \\
\hline Região Norte & 15 & 3,3 & 39 & 8,7 & 188 & 41,9 & 207 & 46,1 & 449 & 100,0 \\
\hline Rondônia & 15 & 28,8 & 34 & 65,4 & 3 & 5,8 & 0 & * & 52 & 100,0 \\
\hline Acre & 0 & * & 0 & * & 3 & 13,6 & 19 & 86,4 & 22 & 100,0 \\
\hline Amazonas & 0 & * & 1 & 1,6 & 26 & 41,9 & 35 & 56,5 & 62 & 100,0 \\
\hline Roraima & 0 & * & 0 & * & 0 & * & 15 & 100,0 & 15 & 100,0 \\
\hline Pará & 0 & * & 0 & * & 28 & 19,6 & 115 & 80,4 & 143 & 100,0 \\
\hline Amapá & 0 & * & 0 & * & 2 & 12,5 & 14 & 87,5 & 16 & 100,0 \\
\hline Tocantins & 0 & * & 4 & 2,9 & 126 & 90,6 & 9 & 6,5 & 139 & 100,0 \\
\hline Região Nordeste & 24 & 1,3 & 49 & 2,7 & 541 & 30,3 & 1.173 & 65,6 & 1.787 & 100,0 \\
\hline Maranhão & 0 & * & 0 & * & 5 & 2,3 & 212 & 97,7 & 217 & 100,0 \\
\hline Piauí & 0 & * & 0 & * & 141 & 63,8 & 80 & 36,2 & 221 & 100,0 \\
\hline Ceará & 0 & * & 0 & * & 17 & 9,2 & 167 & 90,8 & 184 & 100,0 \\
\hline Rio Grande do Norte & 0 & * & 0 & * & 26 & 15,7 & 140 & 84,3 & 166 & 100,0 \\
\hline Paraíba & 0 & * & 0 & * & 14 & 6,3 & 209 & 93,7 & 223 & 100,0 \\
\hline Pernambuco & 1 & 0,5 & 0 & * & 93 & 50,3 & 91 & 49,2 & 185 & 100,0 \\
\hline Alagoas & 0 & * & 0 & * & 89 & 88,1 & 12 & 11,9 & 101 & 100,0 \\
\hline Sergipe & 23 & 30,7 & 49 & 65,3 & 3 & 4,0 & 0 & * & 75 & 100,0 \\
\hline Bahia & 0 & * & 0 & * & 153 & 36,9 & 262 & 63,1 & 415 & 100,0 \\
\hline Região Sudeste & 668 & 40,1 & 639 & 38,4 & 357 & 21,4 & 2 & 0,1 & 1.666 & 100,0 \\
\hline Minas Gerais & 289 & 33,9 & 269 & 31,5 & 293 & 34,3 & 2 & 0,2 & 853 & 100,0 \\
\hline Espírito Santo & 25 & 32,5 & 39 & 50,6 & 13 & 16,9 & 0 & * & 77 & 100,0 \\
\hline Rio de Janeiro & 28 & 30,8 & 57 & 62,6 & 6 & 6,6 & 0 & * & 91 & 100,0 \\
\hline São Paulo & 326 & 50,5 & 274 & 42,5 & 45 & 7,0 & 0 & * & 645 & 100,0 \\
\hline Região Centro-oeste & 49 & 11,0 & 225 & 50,4 & 172 & 38,6 & 0 & * & 446 & 100,0 \\
\hline Mato Grosso do Sul & 1 & 1,3 & 37 & 48,1 & 39 & 50,6 & 0 & * & 77 & 100,0 \\
\hline Mato Grosso & 30 & 23,8 & 68 & 54,0 & 28 & 22,2 & 0 & * & 126 & 100,0 \\
\hline Goiás & 18 & 7,4 & 119 & 49,2 & 105 & 43,4 & 0 & * & 242 & 100,0 \\
\hline Distrito Federal & 0 & * & 1 & 100,0 & 0 & * & 0 & * & 1 & 100,0 \\
\hline Região Sul & 612 & 52,8 & 431 & 37,2 & 116 & 10,0 & 0 & * & 1.159 & 100,0 \\
\hline Paraná & 128 & 32,1 & 224 & 56,1 & 47 & 11,8 & 0 & * & 399 & 100,0 \\
\hline Santa Catarina & 280 & 95,6 & 13 & 4,4 & 0 & * & 0 & * & 293 & 100,0 \\
\hline Rio Grande do Sul & 204 & 43,7 & 194 & 41,5 & 69 & 14,8 & 0 & * & 467 & 100,0 \\
\hline Brasil & 1.368 & 24,8 & 1.383 & 25,1 & 1.374 & 25,0 & 1.382 & 25,1 & 5.507 & 100,0 \\
\hline
\end{tabular}

* Prevalência de $0 \%$.

rança alimentar grave, que variaram de $5,4 \%$ a $22,8 \%$. As diferenças inter-estaduais, porém, já haviam sido documentadas na publicação oficial da própria PNAD, que revelou que enquanto a insegurança alimentar grave acometia $18 \%$ dos domicílios no Maranhão, a mesma situação só era verificada em $2 \%$ daqueles localizados em Santa Catarina 5 .
Dentre as várias explicações para essas disparidades, a desigualdade social apresentada no Brasil é o fator que mais claramente justifica essa situação. Um exemplo é a desigualdade de renda, já evidenciada por Hoffman 18 como o determinante isolado mais importante na ocorrência da insegurança alimentar. A aferição da insegurança alimentar, quando feita pela EBIA, engloba forte influência do componente de acesso financeiro 
ao alimento, uma vez que a maior parte das perguntas da escala refere-se à condição "ter dinheiro suficiente" para a aquisição do alimento ${ }^{10}$. A maior concentração de municípios com insegurança alimentar grave na Região Nordeste pode, então, ser parcialmente explicada por uma maior concentração de pobreza nesta área. Enquanto que no Brasil, 25,3\% da população estão abaixo da linha de pobreza (rendimento menor que $1 / 2$ salário mínimo per capita), no Nordeste este percentual é de $44,9 \%$, quase três vezes maior que o da Região Sul (15,9\%) 19 .

Renda, apesar de sua forte relação com insegurança alimentar, não é suficiente para explicá-la 20. Famílias com renda acima da linha de pobreza podem apresentar insegurança alimentar, pois outras condições podem determinar o acesso ao alimento 21 . Características do responsável pelo domicílio e indicadores do acesso da população a bens e serviços essenciais, como moradia, saneamento e educação também estão intimamente ligados à pobreza e fome 22 . As regiões Norte e Nordeste, que apresentaram maiores médias de insegurança alimentar grave entre seus municípios, são também as que apresentam os piores resultados entre os outros indicadores sociais preditivos da insegurança alimentar grave trabalhados no modelo, como escolaridade. No nordeste, por exemplo, concentra-se a maior parte (52\%) dos 14,1 milhões de analfabetos do país 8 .

As variações intra-estaduais na ocorrência da insegurança alimentar grave nessas duas regiões acompanham as desigualdades sociais experimentadas pelos estados que as compõem. O Maranhão, estado que apresentou a maior variabilidade na ocorrência de insegurança alimentar grave entre seus municípios, é também o com mais desigualdade social, expressa pelo Índice de Desenvolvimento Humano Municipal (IDH-M), renda de 0,636 em 2000, contra 0,822 de Santa Catarina 23 , estado com menor discrepância interna e menores prevalências de insegurança alimentar grave municipal.

A despeito do quadro preocupante apresentado por todas as UF que compõem as regiões Norte e Nordeste, especial atenção deve ser dada ao Estado de Roraima, onde todos os municípios apresentam estimativas muito altas de insegurança alimentar grave. Mais uma vez a pobreza parece ser o principal determinante dessa situação, pois em Roraima 33,4\% das famílias vivem abaixo da linha de pobreza ${ }^{19}$. Vale ressaltar que nesse estado as carências e privações sociais são numerosas e oriundas mais de desigualdades estruturais do que por características individuais 24 , justificando assim essa disseminação da condição em todo o Estado de Roraima.
A intensa ocorrência da insegurança alimentar grave entre os municípios da Região Norte é intrigante, uma vez que a região, por sua localização, favorece a prática extrativista de alimentos 25. Porém, a população é também muito vulnerável à influência de seus ecossistemas, que podem determinar escassez alimentar em tempos de cheias nos rios da região 26 e, por outro lado, impossibilidade de acesso se os rios secarem demais. Interessante notar que nessa dicotomia, a indisponibilidade alimentar prevalece, tornando os moradores mais vulneráveis em relação à insegurança alimentar grave.

Situação interessante, sem dúvida, é a apresentada por Sergipe, que se mostra muito discrepante dos demais estados da Região Nordeste, com $96 \%$ dos seus municípios com estimativas de baixa e média prevalências de insegurança alimentar grave. Essa melhor condição pode ser parcialmente explicada pelo fato desse estado apresentar indicadores socioeconômicos ligeiramente melhores que os demais da região. Em Sergipe, apenas $15,2 \%$ da população estão em situação de miséria (renda $\leq 1 / 4$ salário mínimo per capita), enquanto que na Região Nordeste, em que ele se encontra inserido, esta proporção é de $19,6 \% 19$. Esse melhor desempenho de Sergipe merece ser investigado, uma vez que a renda não é capaz de explicar integralmente esse fenômeno, e os outros indicadores de segurança alimentar e de proteção social, tais como cobertura de programas de transferência de renda e de atenção básica à saúde, não divergem dos demais estados da região 27,28 .

Já nas regiões Sudeste e Sul do país predominam municípios com baixa exposição à insegurança alimentar grave. Na Região Sudeste, somente o Estado de Minas Gerais apresenta municípios com prevalência muito alta de insegurança alimentar grave, sendo que na Região Sul são inexistentes os municípios nesta condição. A pior situação de Minas Gerais frente aos demais estados da sua região pode ser parcialmente explicada pela insegurança alimentar ocorrer em municípios situados na região semi-árida do Brasil, onde a seca é fator determinante para a menor disponibilidade de alimentos 29.

Analisando-se as regiões Sul e Sudeste, observa-se que os menores valores de prevalência de insegurança alimentar grave refletem a melhor situação destas regiões frente às demais, fato explicado pela existência de melhores condições socioeconômicas e ambientais em que vivem a sua população ${ }^{19}$. Tradicionalmente, a Região Sul apresenta os melhores resultados no que se refere à situação de segurança alimentar, o que pode ser observado também por outros indicadores, como, por exemplo, o percentual 
de crianças com baixo peso. Na Região Sul apenas 2,9\% das crianças menores de cinco anos têm déficit de peso, contra 5,2\% na Região Nordeste 30 . Comparação semelhante pode ser realizada em relação à Região Centro-oeste, que tem seus resultados mais próximos à realidade dessas duas regiões do que os das regiões da porção setentrional do Brasil.

Outro estudo com base no mesmo banco de dados da PNAD 2004 indicou que o risco de um domicílio apresentar insegurança alimentar diminuía se a família recebesse algum programa de transferência de renda. A chance de situação de segurança alimentar aumentava em torno de $8 \%$ para cada 10 Reais de acréscimo nos valores das transferências socais. Os resultados obtidos mostram associação positiva da transferência de renda com a segurança alimentar, independentemente do efeito de outras condições 31 . Isso indica que as condições observadas em 2004 , ano em que o Programa Bolsa Família ainda estava em implantação e expansão, podem ter se modificado. Estados como Roraima, que no presente estudo mostrou-se o mais vulnerável à insegurança alimentar grave, segundo dados do Ministério do Desenvolvimento Social e Combate à Fome, já em 2006, tinham 100\% de sua população pobre se beneficiando dos recursos do Bolsa Família, sendo o recurso médio repassado o segundo mais elevado do Brasil 32.

\section{Conclusão}

O estudo permitiu estimar a prevalência insegurança alimentar grave para os 5.507 municípios brasileiros, da malha municipal do Censo Demográfico 2000. As estimativas de insegurança alimentar grave geradas revelam grandes variações intra e inter-estaduais, na sua ocorrência. Existe uma concentração maior de municípios com prevalências elevadas de insegurança alimentar grave nas regiões Norte e Nordeste do Brasil, em contraste com as regiões Sul e Sudeste, que apresentam a maior parte de seus municípios com baixas e médias exposições à insegurança alimentar.

A situação municipal de insegurança alimentar grave desenha uma situação já conhecida de disparidades sociais, há muito mantidas no país. As diferenças regionais, estaduais e municipais são substanciais e resultado da atuação do Estado como provedor de condições propícias à consecução do direito humano à alimentação de sua população. Conclui-se que é importante realçar e analisar as grandes variações inter e intra-regionais na prevalência de insegurança alimentar grave no Brasil, no intuito de subsidiar políticas públicas. 


\section{Resumo}

Estimou-se a prevalência de insegurança alimentar grave para os municipios brasileiros, com base na Pesquisa Nacional por Amostra de Domicílios (PNAD) 2004. Inicialmente, foi gerado e testado um modelo por regressão logística multivariada com base nesse banco de dados. O modelo foi aplicado à amostra do Censo Demográfico de 2000, gerando estimativas de prevalências de insegurança alimentar grave para os municípios brasileiros, que foram analisadas de acordo com o percentual de famílias em condição de insegurança alimentar grave. Essa insegurança alimentar está mais concentrada nas regiões Norte e Nordeste, onde 46,1\% e 65,3\% dos municípios, respectivamente, apresentam altas prevalências de insegurança alimentar grave. Predominam nas regiões Sudeste e Sul municípios com baixa exposição à insegurança alimentar grave. No Centro-oeste a maior parte dos municípios mostra estimativas de insegurança alimentar grave classificadas como médias. Verificou-se grande variação intra-regional na ocorrência da insegurança alimentar, sendo a Região Sul a mais uniforme. Concluise que o Brasil apresenta grandes variações inter e intra-regionais na ocorrência da insegurança alimentar, sendo importante realçá-las e analisá-las, no intuito de subsidiar políticas públicas.

Fome; Previsões; Cidades; Nutrição em Saúde Pública

\section{Colaboradores}

M. B. Gubert, M. H. D’A. Benício e L. M. P. Santos participaram da concepção do trabalho, análises e redação final do artigo.

\section{Agradecimentos}

Pesquisa financiada pelo Conselho Nacional de Desenvolvimento Científico e Tecnológico (CNPq; processo no. 477651/2006-2).

\section{Referências}

1. Pereira RA, Santos LMP. A dimensão da insegurança alimentar. Rev Nutr 2008; 21 Suppl:7s-13s.

2. Castro J. Geografia da fome: o dilema brasileiro: pão ou aço. Rio de Janeiro: Editora Civilização Brasileira; 2001

3. Brasil. Lei $n^{\circ}$. 11.346, de 15 de setembro de 2006. Cria o Sistema Nacional de Segurança Alimentar e Nutricional com vistas em assegurar o direito humano à alimentação adequada e dá outras providências. Diário Oficial da União 2006; 18 set.

4. Pérez-Escamilla R. Experiência internacional com a escala de percepção da insegurança alimentar. In: Ministério do Desenvolvimento Social e Combate à Fome, organizador. Brasília: Ministério do Desenvolvimento Social e Combate à Fome; 2005. (Cadernos de Estudos Desenvolvimento Social em Debate, 2).
5. Instituto Brasileiro de Geografia e Estatística. Pesquisa Nacional por Amostra de Domicílios - suplemento segurança alimentar. Rio de Janeiro: Instituto Brasileiro de Geografia e Estatística; 2006.

6. Segall-Corrêa AM. Insegurança alimentar medida a partir da percepção das pessoas. Estud Av 2007; 21:143-54.

7. Dain S, Soares LT. Reforma do Estado e políticas públicas: relações intergovernamentais e descentralização. In: Oliveira MA, organizador. Reforma do Estado e políticas de emprego no Brasil. Campinas: Universidade Estadual de Campinas; 1998. p. 30-72. 
8. Instituto Brasileiro de Geografia e Estatística. Síntese de indicadores sociais - uma análise das condições de vida da população brasileira. Rio de Janeiro: Instituto Brasileiro de Geografia e Estatística; 2008.

9. Vianna RPT, Segall-Corrêa AM. Insegurança alimentar das famílias residentes em municípios do interior do Estado da Paraíba, Brasil. Rev Nutr 2008; 21 Suppl:111s-22s.

10. Corrêa MAS, Pérez-Escamilla R, Marin-Leon L, Yuyama L, Vianna RPT, Coitinho D, et al. Evaluation of household insecurity in Brazil: validity assessment in diverse sociocultural settings. Santiago: Food and Agriculture Organization of the United Nations; 2008.

11. Elbers C, Lanjouw JOE, Lanjouw P. Micro-level estimation of welfare. Washington DC: World Bank; 2002. (Policy Research Working Paper, 2911).

12. Gubert MB. Modelo preditivo de insegurança alimentar grave para municípios brasileiros [Tese de Doutorado]. Brasília: Universidade de Brasília; 2009.

13. Benicio MHD'A, Venancio SI, Konno SC, Monteiro CA. Novas estimativas para a prevalência de desnutrição na infância nos 5.507 municípios brasileiros a partir de modelos logísticos multinível aplicados à amostra de crianças do Censo 2000. São Paulo: Núcleo de Pesquisas Epidemiológicas em Nutrição e Saúde, Universidade de São Paulo; 2005. (Série Pesquisas em Epidemiologia Nutricional, 1/05).

14. Instituto Brasileiro de Geografia e Estatística. Mapa da pobreza e desigualdade nos municípios brasileiros, 2003. Rio de Janeiro: Banco Mundial/Instituto Brasileiro de Geografia e Estatística; 2008.

15. Benicio MHD, Monteiro CA. Desnutrição infantil nos municípios brasileiros - risco de ocorrência. Brasília: Fundo das Nações Unidas para a Infância/Núcleo de Pesquisas Epidemiológicas em Nutrição e Saúde, Universidade de São Paulo; 1997.

16. Bianchini ZM, Albieri S. Amostragem na coleta dos dados do Censo Demográfico 2000: uma versão resumida. http://www.ibge.gov.br/censo/text amostragem.shtm (acessado em 18/Mai/2010).

17. Silva PLN, Pessoa DGC, Lila MF. Análise estatística de dados da PNAD: incorporando a estrutura do plano amostral. Ciênc Saúde Coletiva 2002; 7 : 659-70.

18. Hoffmann R. Determinantes da insegurança alimentar no Brasil. Análise dos dados da PNAD de 2004. Revista Segurança Alimentar e Nutricional 2008; 15:49-61.

19. Instituto Brasileiro de Geografia e Estatística. Pesquisa Nacional por Amostra de Domicílios. Volume Brasil. Rio de Janeiro: Instituto Brasileiro de Geografia e Estatística; 2008.

20. Rose D. Economic determinants and dietary consequences of food insecurity in the United States. J Nutr 1999; 129 Suppl:S517-20.
21. Panigassi G, Segall-Corrêa AM, Marin-León L, Pérez-Escamilla R, Sampaio MFA, Maranha LK. Insegurança alimentar como indicador de iniqüidade: análise de inquérito populacional. Cad Saúde Pública 2008; 24:2376-84.

22. Monteiro CA. A dimensão da pobreza, da fome e da desnutrição no Brasil. Estud Av 1995; 9:195207.

23. Programa das Nações Unidas para o Desenvolvimento. Atlas do desenvolvimento humano no Brasil. Rio de Janeiro: Instituto de Pesquisa Econômica Aplicada/Fundação João Pinheiro; 2003.

24. Drachler ML, Côrtes SMV, Castro JD, Leite JCC. Proposta de metodologia para selecionar indicadores de desigualdade em saúde visando definir prioridades de políticas públicas no Brasil. Ciênc Saúde Coletiva 2003; 8:461-70.

25. Yuyama LKO, Aguiar JPL, Pantoja L, Maeda RN, Melo T, Alencar FH, et al. Segurança/Insegurança Alimentar em famílias urbanas e rurais no Estado do Amazonas: I. Validação de metodologia e de instrumento de coleta de informação. Acta Amaz 2007; 37:247-52.

26. Alencar FH, Yuyama LKO, Varejão MJC, Marinho HA. Determinantes e conseqüências da insegurança alimentar no Amazonas: a influência dos ecossistemas. Acta Amaz 2007; 37:413-8.

27. Instituto Brasileiro de Geografia e Estatística. Pesquisa de Orçamentos Familiares - análise da disponibilidade domiciliar de alimentos e do estado nutricional no Brasil. Rio de Janeiro: Instituto Brasileiro de Geografia e Estatística; 2004.

28. Ministério do Desenvolvimento Social e Combate à Fome. MDS em números. http://www.mds. gov.br/sites/mds-em-numeros/ (acessado em 19/ Mai/2010).

29. Secretaria de Avaliação e Gestão da Informação, Ministério do Desenvolvimento Social e Combate à Fome. Cadernos de estudos desenvolvimento social em debate, $n^{\circ}$. 4. Brasília: Ministério do Desenvolvimento Social e Combate à Fome; 2006.

30. Ministério da Saúde. Pesquisa Nacional de Demografia e Saúde da Criança e da Mulher - PNDS 2006: dimensões do processo reprodutivo e da saúde da criança. Brasília: Centro Brasileiro de Análise e Planejamento/Ministério da Saúde; 2009.

31. Segall-Corrêa AM, Marin-Leon L, Helito H, PérezEscamilla R, Santos LMP, Paes-Sousa R. Transferência de renda e segurança alimentar no Brasil: análise dos dados nacionais. Rev Nutr 2008; 21 Suppl:39s-51s.

32. Secretaria Nacional de Renda e de Cidadania, Ministério do Desenvolvimento Social e Combate à Fome. Informações do cadastro único. http://www.mds.gov.br/adesao/mib/matri zviewuf.asp?UF=RR (acessado em 07/Mai/2010).

Recebido em 03/Fev/2010

Versão final reapresentada em 19/Mai/2010

Aprovado em 21/Jun/2010 